\title{
SUPERLUMINAL MOTION IN CTA 102
}

\author{
ANN E. WEHRLE \\ Owens Valley Radio Observatory \\ California Institute of Technology \\ Pasadena, California 91125 \\ $U S A$
}

Sholomitskii (1965) discovered that the flux density of the quasar CTA 102 varies at low frequencies on a timescale of a few months. Low-frequency variability can be explained by "superluminal flux variation" (Romney et al. 1984): If the intrinsic brightness of a component moving in a relativistically beamed source varies by only a few percent, the observer sees its flux density change by a much larger factor $\delta^{3-\alpha}$ when the optically thin blob moves almost directly toward the observer. Such a relativistically beamed source is likely to exhibit superluminal motion if studied with sufficient resolution and sensitivity. Superluminal motion in CTA 102 was discovered by Båăth (1987) who concluded on the basis of maps made at three epochs at a frequency of $932 \mathrm{MHz}$ that two components were separating at a rate of 0.65 milliarcseconds (mas) per year. Using a redshift $z=1.037$ and $H_{0}=100 \mathrm{~km} \mathrm{~s}^{-1} \mathrm{Mpc}^{-1}, q_{0}=0.5$, this expansion speed corresponds to $(18 \pm 4) h^{-1} c$. The extraordinarily high speed led us to make VLBI images of the source at a higher frequency in order to increase the resolution and make a more precise determination of the speed.

The observations were conducted at $5 \mathrm{GHz}$ with 11 antennas of the United States and European VLBI Networks with the addition of an antenna in Hartebeesthoek, South Africa. The observations occurred in June 1987 and in June 1988. The data were processed on the Caltech/JPL correlator. The highest resolution achieved was 1 mas.

A preliminary image of CTA 102 is shown in the figure. The source displays very unusual complex morphology: at least four components, enveloped by a low-surface-brightness "cocoon", form a curved structure. We expect to identify the core when similar data at $1.7 \mathrm{GHz}$ are reduced.

CTA 102 contains the fastest superluminal component of any VLBI source known to date (Bååth 1987). The components may be whirling along a helix or sliding along a straight path until they hit a "brick wall". Twisted motion of components in a jet has been convincingly demonstrated in 3C 273 (Zensus et al. 1988), and in 3C 345 (the Caltech group, in preparation). If the superluminal speed derived by Båăth is correct for the $6-\mathrm{cm}$ components, we should be able to observe signs of this "twisted" motion in just a few years. Monitoring the source for a few years and modelling the components as several planar or conical shocks moving in a jet may clarify the ejection orientation and gamma factor of the relativistically beamed source.

This work was supported in part by the National Science Foundation under grant AST 85-09822. 


\section{References}

Bååth, L. B. 1987, in Zensus and Pearson 1987, p. 206.

Romney, J. D., Alef, W., Pauliny-Toth, I. I. K., Preuss, E., and Kellermann, K. I. 1984 in IAU Symposium 110, VLBI and Compact Radio Sources ed. R. Fanti, K. Kellermann and G. Setti (Dordrecht:Reidel).

Sholomitskii, G. B. 1965 English translation in Soviet Astron., 9, 516.

Zensus, J. A., Bååth, L. B., Cohen, M. H., and Nicolson, G. D. 1988, Nature, 334, 410.

Zensus, J. A., and Pearson, T. J. (eds.) 1987, Superluminal Radio Sources (Cambridge: Cambridge University Press).

PRELIMINARY IMAGE OF CTA 102: JUNE 1988 CTA $102 \quad 4.990 \mathrm{GHz} 06 / 11 / 88$

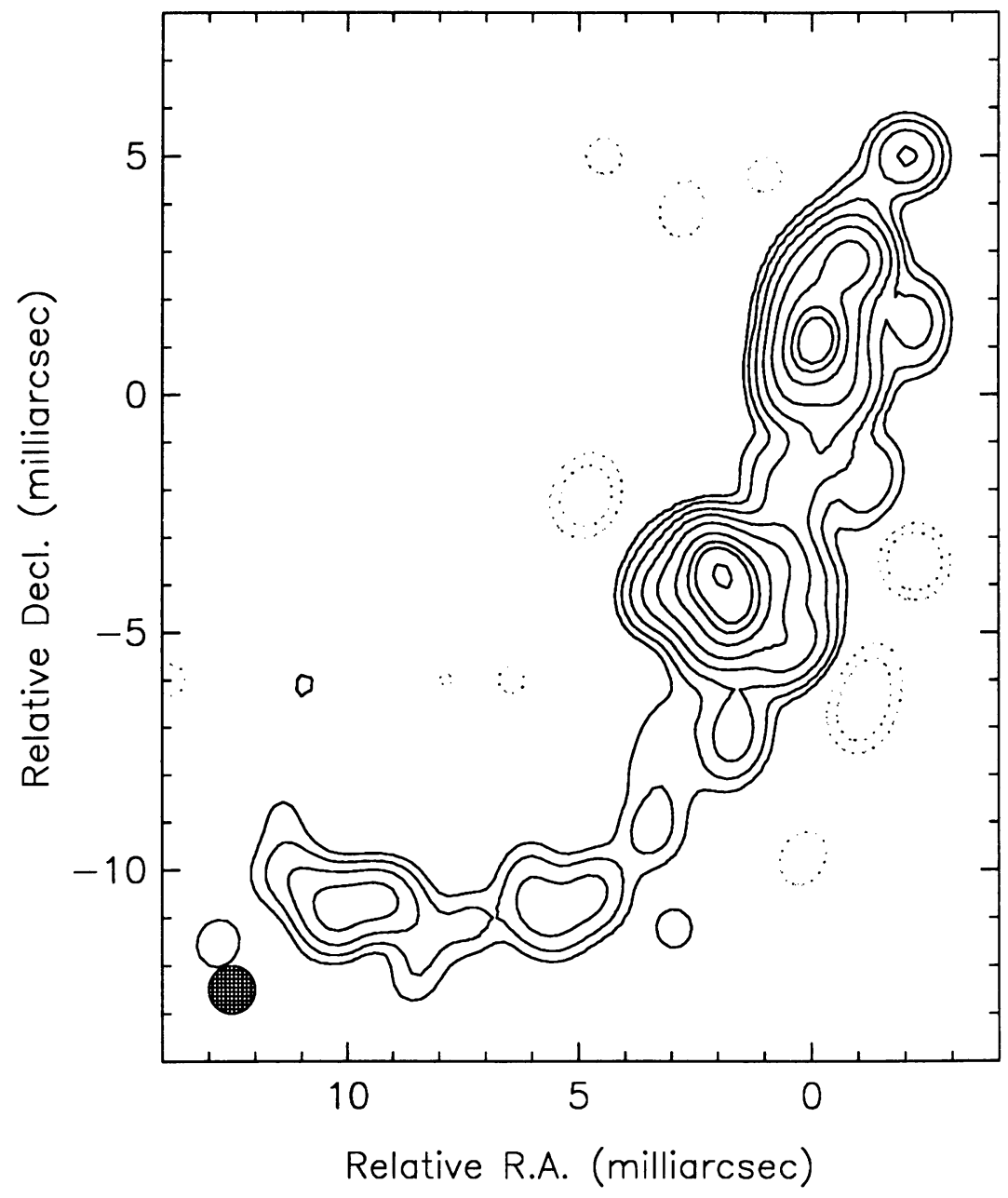

